\title{
Association between birth weight and neuromotor performance: A twin study
}

\author{
A. A. T. Lopes ${ }^{1}$, Go Tani' ${ }^{2}$ P. T. Katzmarzyk ${ }^{3}$, M. A. Thomis ${ }^{4}$, J. A. R. Maia ${ }^{5}$ \\ ${ }^{1}$ Porto Center Hospital and School of Allied Health Technology (ESTSP), Porto Polytechnic Institute, Porto, Portugal, ${ }^{2}$ School of \\ Physical Education and Sport, University of São Paulo, São Paulo, Brazil, ${ }^{3}$ Pennington Biomedical Research Center, Louisiana State \\ University System, Baton Rouge, LA, USA, ${ }^{4}$ Physical Activity, Sports \& Health Research Group, Department of Kinesiology, Faculty of \\ Kinesiology and Rehabilitation Sciences, Katholieke Universiteit Leuven, Leuven, Belgium, ${ }^{5}$ CIFI2D, Faculty of Sports, University of \\ Porto, Porto, Portugal \\ Corresponding author: Mr Alexandre Lopes, MSc, Porto Center Hospital and School of Allied Health Technology (ESTSP), Porto \\ Polytechnic Institute, Avenida António Domingues dos Santos, 231. Rch, Matosinhos 4460-237, Porto, Portugal. E-mail: \\ alexandrelopes5@yahoo.com
}

Accepted for publication 15 July 2013

Studies have shown important associations between low birth weight (BW), a variety of morbidities, and reduced motor performance. Using a twin sample, this study aimed to verify (a) the magnitude of the association between BW and neuromotor performance (NMP); (b) if the NMP of twins is within the normal range; and (c) if monozygotic (MZ) and dizygotic (DZ) twins' intra-pair similarities in NMP are of equal magnitude. We sampled 191 twins (78 MZ; $113 \mathrm{DZ}$ distinguished through their DNA), aged $8.9 \pm 3.1$ years with an average $B W$ of $\mathbf{2 2 4 6 . 3} \pm \mathbf{4 8 5 . 4}$ g; gestational characteristics and sports practices were also assessed. The Zurich Neuromotor test battery, comprising five main tasks, was used; Twins NMP assessments were highly reliable (intra-rater reliability: 0.76-0.99). BW accounted for up to $11 \%$ of the total variance of NMP across the zygosity groups. Between $32.7 \%$ and $76.9 \%$ of children were below the 10th percentile for tasks requiring timing of performance (purely motor task, adaptive fine motor task, dynamic, and static balance), while less than $6.4 \%$ of children were below the 10th percentile for associated movements. MZ twins NMP intraclass correlations showed greater similarity than DZ twins in three of the five tasks, suggesting the importance of genetic factors in NMP.
In recent decades, the development of neonatal intensive care led to an increased prevalence of children with low birth weight (LBW) (Russell et al., 2007), defined by the World Health Organization as weight at birth of less than $2500 \mathrm{~g}$ (5.5 lbs). Based on epidemiological observations, infants weighing less than $2500 \mathrm{~g}$ are approximately 20 times more likely to die than heavier babies. About 20 million infants, or about $23.8 \%$ of all births, are born each year at a LBW. Nearly 4 million babies die in the first month of life and LBW and premature birth are major causes (Wardlaw et al., 2004).

Infants undergoing such intensive care may also be at risk for increased morbidity over the longer term. Several cognitive and neuromotor deficits are prevalent in LBW children. Much of the research to date has focused on those infants born extremely premature (23-28 weeks gestation) or at a very low $(<1500 \mathrm{~g})$ or extremely LBW $(<1000 \mathrm{~g})$ with the highest rates of mortality and morbidity (Msall \& Tremont, 2002; Wolf et al., 2002). Over recent years, there has been an increased interest on the early outcomes of the latepreterm subgroup of premature infants. Late-preterm infants are defined as those born between 34 and 36 6/7 weeks gestation and account for up to 6.9/1000 of all preterm, strongly support the assertion that late preterm infants have higher risks for mortality and morbidity compared with term infants (37 completed weeks through 42 completed weeks) (Escobar et al., 2006a). Because the latter may imply that these infants are almost term and mature, there is the possibility of underestimating their risks, less diligent evaluation, and poor follow-up.

Children with LBW show deficits in distinct domains such as mental retardation, blindness, communication problems, self-care, social level, limitations in motor function, growth disorders, perception, attention, and restrictions in physical activity (Wolf et al., 2002; Wardlaw et al., 2004). However, there is no absolute consensus about its extent and significance. Motor coordination problems, developmental delay, learning disorders, emotional problems, and hyperactivity have been documented, mainly in very LBW (Saigal et al., 1990, 2001; Hack et al., 2000; Palta et al., 2000; Walther et al., 2000; Rogers et al., 2005). Despite the absence of major problems in their neurodevelopment, children with LBW demonstrate "below average" aptitudes in various 


\section{Lopes et al.}

domains, especially level of education, social interaction, and motor skills, hence, substantial recent research has been directed toward understanding long-term problems of LBW children. Thus, it is plausible to propose that long-term morbidity may indeed be a reality and that general developmental immaturity may persist in LBW children.

It has been reported that children from multiple births (e.g., twins) have higher rates of cerebral palsy and neurodevelopment problems when compared with singletons (Hajnal et al., 2005). Twin magnetic resonance imaging studies have found that genetic factors strongly influence several aspects of brain structure, such as cortical thickness, and gray and white matter volumes, where monozygotic twins (MZ) showed higher similarities in the intra-class correlation maps than dizygotic twins (DZ) (Brun et al., 2009). This population of children and adolescents has not been frequently studied with respect to intra-pair similarities in their neuromotor development, and the magnitude and significance of MZ-DZ twin differences in similarities remains largely unknown. The importance of investigating children's motor performance to assess whether their neuromotor development is within the range of normal development has been recently emphasized (Largo et al., 2003). Thus, the present study addresses the associations between birth weight and neuromotor performance using a twin sample. The research questions are: (a) Is there a significant association between birth weight and neuromotor performance? (b) Is the neuromotor performance of twins within the normal range? and (c) Are intra-pair similarities in neuromotor development of MZ and DZ twins of unequal magnitude?

\section{Material and methods}

\section{Sample and general procedures}

The sample was selected from birth records (1990-2002) documented by S. António General Hospital in Porto, and Guimarães General Hospital in Guimarães situated both in the north of Portugal. All twins who had their birth weight in their registry were included in this study; furthermore, problematic twin/multiples pregnancies were not medically managed in these hospitals. The study was approved by the ethics committees of each hospital and written informed consent was obtained from each subject (informed assent) and their parents.

Children were evaluated in the Department of Physical Medicine of each hospital between January and April 2007, and children suffering from cerebral palsy and/or mental retardation (1 child) were excluded. From the 170 contacted families, we assessed 83 pairs of twins, 7 sets of triplets and 1 set of quadruplets, aged 5-17 years; 94 were male and 97 female, and 78 were $\mathrm{MZ}$ and 113 were DZ twin members. On average, their birth weight was $2246.3 \pm 485.5 \mathrm{~g}$. It is expected that triplets and especially quadruplets, have different birth characteristics of the twins, and maybe later in their life histories. In the present study, their birth weight was not very different from the twins, and we did not find any relevant impact on the final results.

Weight and height were measured with the aid of a Joffre scale and a ruler. All children were measured barefoot. Each child was measured individually in the presence of a parent. Furthermore, the set of tasks that each child would have to perform was demonstrated by the same investigator. Initially the child was asked to carry out small activities such as writing, brushing teeth and paper cutting to verify his/her laterality or dominance, and all tasks were first carried out with the dominant side and then with the non-dominant side (Largo et al., 2002). During the assessment, the parents answered a semi-structured questionnaire regarding gestational length, birth weight, birth length, medical problems, Apgar (skin color/ complexion, pulse rate, reflex irritability, muscle tone and breathing) first minute, Apgar fifth minute, which were read from each child health bulletin. In addition, the children's current sports participation (number of years) was reported by all parents. No specific neurological assessment was made, given that the Apgar scores in all twins were 8 or upper which means good health.

\section{Zurich Neuromotor Assessment (ZNA)}

The ZNA was developed by the Center for Growth and Development, University Hospital of Zurich (Largo et al., 2002). This test battery was chosen because it (a) has consistently been tested with regard to reliability; (b) has standardized references; (c) differentiates between motor performance and movement quality; and (d) is based on age and gender specific norms. It was built on different motor tasks (see Table 1) and considers timed performance and quality of movement (associated movements of the contralateral extremity, ipsilateral, face, head, and trunk). The ZNA has validity because it is an instrument that is reliable in implementation, but also records the behavior for which it was developed. The neuromotor examination discriminates between children whose everyday activities are impaired by dysfunction and children whose function is normal (Largo et al., 2001).

The ZNA consists of tasks of varying complexity. The complexity increases from repetitive movements, to alternating movements and sequential movements, and finally to an adaptive task, such as the pegboard. The complexity of the tasks contributes significantly to the differences observed in development and interindividual variability. The ZNA allows a quantitative judgment of motor abilities with regard to timed performance and quality of movements in children aged 5.0-18.5 years. In addition, pure motor and adaptive tasks can be evaluated separately; differences between the upper and lower extremities and difference between dominant and non-dominant sides can be reliably assessed.

Previously reported reliability of measurements of timed performance and ratings of associated movements for tasks of the ZNA on average, for intra-rater and inter-rater reliability were 0.95 and 0.90 for timed performance, and approximately 0.80 and 0.70 for associated movements. Some motor tasks (sequential movements of fingers, static, and dynamic balance) are performed with increasing difficulty depending on the age of the

Table 1. Tasks of Zurich Neuromotor Assessment

\begin{tabular}{ll}
\hline Repetitive movements & $\begin{array}{l}\text { Finger } \\
\text { Hand } \\
\text { Foot } \\
\text { Hand } \\
\text { Fiternating movements }\end{array}$ \\
$\begin{array}{l}\text { Foot } \\
\text { Sequential movements }\end{array}$ & Fingers \\
$\begin{array}{l}\text { Pegboard } \\
\text { Dynamic balance }\end{array}$ & Sideward \\
$\begin{array}{l}\text { Static balance } \\
\text { Stress gaits }\end{array}$ & Walking on the toes \\
& Walking on the heels \\
& Walking on the outer soles of feet \\
& Walking on the inner soles of feet
\end{tabular}




\section{Neuromotor performance in twins}

children. Motor tasks consist of repetitive movements of the foot (20 per foot), hand (20 per hand), movements of fingers (20 per hand), alternating movements of hands and feet (10 by 10 feet and by hand), and sequential movements of fingers (three sequences by hand). The adaptive tasks require the integration of other sensory systems (visual and tactile). Twelve pins have to be inserted on a pin board with the dominant and nondominant hand. The dynamic balance consists of double jumps forward and sideward. The static balance on one foot at a time is also assessed (Largo et al., 2002).

All assessments were recorded on video (Sony $50 \mathrm{~Hz}$, Sony, New York, NY, USA). Timed performance (speed of movement) was measured using stopwatch with an accuracy of tenths of a second. The exact moment of the beginning of the measurement of time and number of movements were well established in the manual of ZNA to evaluate each motor task. The quality of the movement was scored from associated movements. Associated movements are involuntary movements in parts of the body that are not directly involved in the task. The lower the frequency and exuberance of associated movements the greater the quality of movement. Associated movements are judged according to their frequency and degree.

During each unilateral task, the frequency of associated movements was recorded in tenths of the number of active movements $(0-10)$. For the degree of associated movements, the most exuberant or pronounced throughout the task (value of 0-3) was recorded.

The ZNA is expressed in the form of deviations from the average of the reference population, e.g., in $z$-scores according to age and sex. The ZNA software analyzes the performance of each subject and assigns $z$-values and percentile ranks to each individual performance and compares them with the "normality band" that ranges from $\mathrm{P}_{10}$ to $\mathrm{P}_{90}$. The evaluation was conducted in components per block (time of performance and associated movements) rather than differential components.

\section{Zygosity determination}

A blood sample was collected from each twin member, and the extraction of DNA was performed with a method based on the use of Chelex resin. Genotyping was performed on an ABI 310 Genetic Analyzer (AB Applied Biosystems, Life Technologies Europe BV, Porto, Portugal), according to the manufacturer's instructions, for determining the size of DNA fragments and comparison with allelic scales provided with commercial kits. The automatic determinations of the size of specific fragments were amplified by polymerase chain reaction (PCR) for highly polymorphic loci (e.g., micro-satellites). In all DNA samples, the analysis of 17 short tandem repeat (STRs) autosomal (CSF1PO, D2S1338, D3S1358, D5S818, D7S820, D8S1179, D13S317, D16S539, D18S51, D19S433, D21S11, alpha fibrinogen, pentanucleotides, TH01, thyroid peroxidase, and von Willebrand factor) and the Amelogenin locus (sex determination) was performed by PCR amplification, using commercial kits Powerplex 16 System (Promega Corporation, Fitchburg, WI, USA) and Identifiler (AB Applied Biosystems), according to the manufacturer's instructions. Allele frequencies of the different genetic markers in the north and center of Portugal were used in the computation of probabilities of being MZ (Lareu et al., 1994).

To ascertain data quality of the neuromotor performance scores, intra- and inter-rater reliability was verified. Video recordings of 10 children were randomly chosen. Each of the three observers scored the 10 children. After 1 week, each observer re-examined their assessments in a random sequence of the 10 children. For time performance and quality of movement in all tasks, the analysis of variance-based intraclass correlation coefficients (ICC) varied from 0.79 to 0.99 (intra-rater), and from 0.76 to 1.00 (interobserver). In associated movements ICC varied from 0.80 to 0.99 , and inter-observer from 0.80 to 0.98 .

\section{Statistical procedures}

Exploratory and descriptive analysis was conducted for each variable. When dealing with limited twin sample sizes, it has been suggested (Bouchard et al., 1997), to increase statistical power, to analyze only MZ and DZ twins controlling for such covariates as their age, age $^{2}$, and sex, and in our case, also for sports participation using forward multiple regression. Thus, our final phenotypes for all ZNA performance tests were the standardized residuals from such an analysis. Using these residuals, regression models were further used to identify the importance of birth weight (Model 1), and birth weight plus gestational age and Apgar (Model 2) in neuromotor performance within each zygosity.

ICC (denoted here as $t$ ) were used to describe the within pair homogeneity in the residuals of their neuromotor performance. MZ twins are expected to have higher $t$-values than DZ twins when genetic factors contribute to interindividual differences in ZNA. Differences between $t_{\mathrm{MZ}}$ and $t_{\mathrm{DZ}}$, bootstrap estimates of its standard errors, and $z$-tests were calculated in STATA 10 (College Station, TX, USA). Statistical significance was set at 5\%.

\section{Results}

Table 2 presents basic descriptive characteristics of the sample. No statistically significant mean differences $(P>0.05)$ were found between the descriptive characteristics of boys and girls and MZ and DZ twins. On average, twins had LBW $(<2500 \mathrm{~g})$ and were preterm ( $<36$ weeks). As expected, Apgar scores are lower at 1 min when compared with 5 min. Most twins played sports $(>70.2 \%)$ and have a right dominant side $(>80.4 \%)$.

In Table 3 the explained variance is provided for covariates (age, age ${ }^{2}$, sex, and sport participation combined) for all neuromotor tasks in all twin pairs irrespective of their zygosity (Model 0). Values are low, $7 \%$ in adaptive fine motor tasks, to moderate, $26 \%$ in purely motor tasks. The only exception was for dynamic balance where the combination of these covariates did not account for any of the variability present in twin performance.

Table 3 shows results of explained variance by birth weight (Model 1), birth weight, gestational age and Apgar at 5-min scores combined (Model 2) in the standardized residuals of the five neuromotor performance tasks. With the exception of dynamic balance, birth weight explained $7-15 \%$ of the variance of neuromotor tasks in MZ twins; in DZ twins explained variance is $1-3 \%$ in three tests, and zero in two of them (dynamic balance and associated movements). When gestational age and Apgar scores at $5 \mathrm{~min}$ were incorporated in the regression equations (Model 2), 26\% of the variance in adaptive fine motor task was explained in MZ twins, whereas the lowest $R^{2}$ was for associated movements (6\%). In DZ twins, no substantial increase in explained variance is found regarding model 0 .

The percentage of children with neuromotor performance lower than $\mathrm{P}_{10}$ was high, between $32.7 \%$ and $76.9 \%$, for tasks requiring timing of performance (purely motor task, adaptive fine motor task, dynamic, and static balance). For associated movements percentages were 
low, between $2.6 \%$ and $6.4 \%$. Overall, DZ twins have similar frequencies (percentages) compared with $\mathrm{MZ}$ twins for all neuromotor items (Table 4).

In Table $5 \mathrm{t}$ values for MZ and DZ pairs in each task, their differences and corresponding test statistics are reported. Intra-pair similarities were higher for $\mathrm{MZ}$ twins compared with DZ twins for purely motor tasks, adaptive motor tasks, and borderline higher for dynamics balance. For the other neuromotor tasks no significant differences were found between the zygosity groups.

\section{Discussion}

Assessing and estimating neuromotor performance in children and adolescents is a time-consuming and precise task. Notwithstanding this difficulty, this study aimed to investigate birth weight effects on $\mathrm{MZ}$ and $\mathrm{DZ}$ twins' neuromotor performance, if their performance was within the normal range, and if neuromotor performance was similar in twins of different zygosity. In very general terms, the results showed that birth weight had a limited influence on neuromotor performance; furthermore, there is greater homogeneity in neuromotor performance in $\mathrm{MZ}$ than in $\mathrm{DZ}$ twins.

In the present report, gestational weight and age are, on average, $2246.3 \pm 485.5 \mathrm{~g}$ and $35.2 \pm 2.2$ weeks, respectively, and often minor neurological signs are associated with developmental coordination disorders (Escobar et al., 2006a, b). No subjects from this sample were referenced to medical problems or specialized monitoring. Furthermore, the birth APGAR score was not lower than 8 , which is considered good health. These facts pose some difficulty when assessing and comparing the present results with those from other studies because our MZ and DZ twin sample did not include any subjects with a birth weight less than $1000 \mathrm{~g}$. Neuromotor performance may be conditioned, to some extent, by the twins' birth weight, with those twins born with LBW performing worst. Therefore, we included late-preterm infants defined by birth at 35 through 36 weeks' gestation in the low birth twins since they are less physiologically and metabolically mature than term infants (Escobar et al., 2006a). Of importance is the fact that a birth weight of $>2000 \mathrm{~g}$ is not protective against assisted ventilation or readmission and the use of birth weight as a predictor remains quite common, in both the literature and clinical practice. However, much less is known about premature infants at higher gestations. It is clear from the limited literature that late preterm infants, however defined, experience greater mortality and morbidity than term infants. These considerations highlight the need to devote more research attention to late preterm infants, although they do not experience as much morbidity as very premature infants, their numbers are much greater. Eight percent of the late preterm infants require supplemental oxygen support for at least $1 \mathrm{~h}$, almost 3 times the rate found in infants born at $>37$ 
Neuromotor performance in twins

Table 3. Explained variance for Model 0 (covariates include age, age ${ }^{2}$, sex and sports participation combined in all twin pairs for each neuromotor task); Model 1 (uses only birth weight) and Model 2 (birth weight, plus gestational age and Apgar score at $5 \mathrm{~min}$ ) in the five neuromotor performance tasks

\begin{tabular}{|c|c|c|c|c|c|}
\hline \multirow[t]{2}{*}{ Tasks } & \multirow{2}{*}{$\frac{\text { Model } 0(\%)}{\text { All twin pairs }}$} & \multicolumn{2}{|c|}{ Model $1^{*}(\%)$} & \multicolumn{2}{|c|}{ Model 2* (\%) } \\
\hline & & MZ & $\mathrm{DZ}$ & MZ & $\mathrm{DZ}$ \\
\hline Purely motor tasks & 26.0 & 11.0 & 1.0 & 14.0 & 1.0 \\
\hline Adaptive fine motor task & 7.0 & 15.0 & 3.0 & 26.0 & 3.0 \\
\hline Dynamic balance & 0.0 & 0.0 & 0.0 & 9.0 & 1.0 \\
\hline Static balance & 13.0 & 8.0 & 2.0 & 9.0 & 5.0 \\
\hline Associated movements & 16.0 & 7.0 & 0.0 & 6.0 & 0.0 \\
\hline
\end{tabular}

*Models 1 and 2 are predicting the standardized residuals obtained from model 0. DZ, dizygotic; MZ, monozygotic.

Table 4. Absolute frequencies and percentages of children with neuromotor performance scores below "normal" $(<\mathrm{P} 10)$ cut-off scores

\begin{tabular}{lccc}
\hline Tasks & Total $N(\%)$ & MZ N (\%) & DZ N (\%) \\
\hline Purely motor tasks & $130(68.4)$ & $47(61.0)$ & $83(73.4)$ \\
Adaptive fine motor tasks & $136(71.5)$ & $58(75.3)$ & $78(69.0)$ \\
Dynamic balance & $137(72.1)$ & $50(64.9)$ & $87(76.9)$ \\
Static balance & $63(33.1)$ & $26(33.7)$ & $37(32.7)$ \\
Associated movements & $8(4.2)$ & $5(6.4)$ & $3(2.6)$ \\
\hline
\end{tabular}

DZ, dizygotic; MZ, monozygotic.

weeks. Following discharge from neonatal hospitalization, late preterm infants were much more likely to be re-hospitalized than term infants, and this increase was evident both within 14 days as well as within 15 to 182 days after discharge, however, there are no studies on this relationship with motor performance (Escobar et al., 2006b).

Age, age ${ }^{2}$, sex, and sports participation explain different amounts of the total variance in neuromotor performance in the total twin group: $26 \%$ (purely motor tasks), 13\% (static equilibrium balance), $7 \%$ (adaptive fine motor task), and $16 \%$ in associated movements. The effect of birth weight only on neuromotor performance accounted for $15 \%$ and $3 \%$ in the adaptive fine motor task in $\mathrm{MZ}$ and DZ respectively. Birth weight explained $11 \%$ and $1 \%$ respectively in $\mathrm{MZ}$ and DZ twins for purely motor tasks, $8 \%$ and $2 \%$ in static balance, $7 \%$ of variance in associated movements in MZ twins only. Overall, birth weight was not or only minimally related to neuromotor performance in the $\mathrm{DZ}$ twins. When using the joint additive effects of birth weight, Apgar $5^{\prime}$ and gestational age, the amount of variance explained was just merely $26 \%$ in the adaptive fine motor task in $\mathrm{MZ}$ twins and $6 \%$ in associated movements. Again in DZ twins we observed extremely low proportions of explained variance.

Other studies have focused their efforts on different deficits. For example, one study using a small Portuguese twin sample aged 6-12 years investigated the importance of genetic factors in explaining physical activity levels and health-related physical fitness performance variability, found that the explained variance by the joint effects of sex and age were around $1-21 \%$ in MZ, and 2-13\% in DZ (Maia et al., 2003). Furthermore, another study examined the association between birth weight and adult body composition, in particular lean body mass, subcutaneous fatness, and fat distribution, in female twins. When the twins were considered as individuals in the analyses, the twins who were heavier at birth were taller and slightly heavier as adults than the lighter co-twins. The authors therefore suggested that the intrauterine environment was critical for the attained adult height (Loos et al., 2002). The hypothesis of prenatal programming (Barker, 1997; Morley et al., 2003) is one of the most credible explanations for the association between birth weight and health. It is usually suspected that the process in which the stimulus or aggression (against the constraints of space and nourishing supplies) experienced by the fetus at critical developmental periods would have repercussions on the structure, organs' role, organ systems, and tissues. Some authors, for example, reported in LBW children of 6 years major motor deficits; if their birth weight was below $1000 \mathrm{~g}$ these children showed impairments of $9 \%$, while those above $1500 \mathrm{~g}$ demonstrate deficits of $4 \%$ (Jongmans et al., 1997). Similarly, another study using a sample of 17 year-old children indicated that those born extremely underweight, e.g., $<800 \mathrm{~g}$, had $55 \%$ of motor deficits in rhythm and cadence (Rogers et al., 2005). These extreme and unfortunate outcomes are far away from those reported in the present study because $\mathrm{MZ}$ and DZ twins did not include any subject with birth weight less than $800 \mathrm{~g}$.

The neuromotor performance of $\mathrm{MZ}$ and $\mathrm{DZ}$ twins in the present study was below normal values $\left(<\mathrm{P}_{10}\right)$. Except for the associated movement scores, high frequencies (32.7-76.9\%) of twins below the $\mathrm{P}_{10}$ value were found for the different neuromotor components. Only one study (Seitz et al., 2006) assessed children (mean age of six years) of birth weight $<1250 \mathrm{~g}$ with the ZNA test battery and found that the components related to execution times of the tasks was below average, between $18 \%$ and $38 \%$. For the associated movements, twins in the current study not only showed values above the reference group, but also produced better results than the study of Evensen et al. (2004) with values of $68 \%$.

Our results showed that there is greater homogeneity in neuromotor performance in MZ than in DZ twins, 


\section{Lopes et al.}

Table 5. Intraclass correlations $(t) \pm$ standard-errors, observed correlation differences (diff), bootstrap standard-errors (bse) for differences between $t_{\mathrm{Mz}}$ and $t_{\mathrm{Dz}}, z$ statistic and corresponding $P$-values

\begin{tabular}{|c|c|c|c|c|c|c|}
\hline Task & Zygosity & $t \pm \mathrm{SE}$ & Diff & Bse & $z$ & $P$-value \\
\hline Purely motor tasks & $\begin{array}{l}\text { MZ } \\
\mathrm{DZ}\end{array}$ & $\begin{array}{l}0.75 \pm 0.07 \\
0.56 \pm 0.09\end{array}$ & 0.19 & 0.08 & 2.37 & 0.02 \\
\hline Adaptive fine motor task & $\begin{array}{l}\mathrm{MZ} \\
\mathrm{DZ}\end{array}$ & $\begin{array}{l}0.65 \pm 0.09 \\
0.38 \pm 0.11\end{array}$ & 0.27 & 0.09 & 2.74 & 0.01 \\
\hline Dynamic balance & $\begin{array}{l}\mathrm{MZ} \\
\mathrm{DZ}\end{array}$ & $\begin{array}{l}0.66 \pm 0.09 \\
0.46 \pm 0.10\end{array}$ & 0.20 & 0.11 & 1.85 & 0.06 \\
\hline Static balance & $\begin{array}{l}\mathrm{MZ} \\
\mathrm{DZ}\end{array}$ & $\begin{array}{l}0.43 \pm 0.13 \\
0.42 \pm 0.11\end{array}$ & 0.01 & 0.15 & 0.05 & 0.96 \\
\hline Associated movements & $\begin{array}{l}\mathrm{MZ} \\
\mathrm{DZ}\end{array}$ & $\begin{array}{l}0.42 \pm 0.13 \\
0.41 \pm 0.11\end{array}$ & 0.01 & 0.21 & 1.13 & 0.26 \\
\hline
\end{tabular}

DZ, dizygotic; MZ, monozygotic.

referring to at least a partial genetic component to explain interindividual variation in LBW children's neuromotor performance. MZ twin similarity was significantly higher than DZ twin similarity for Purely Motor Tasks and Adaptive Fine Motor Task scores (borderline for dynamic balance). This finding is in concordance with a study of Akerman and Fischbein (1992) in which 145 twin pairs up to 18 years were evaluated on locomotor's, social, communication, and motor coordination and performance functions. They noted intrapair similarities in the $\mathrm{MZ}$ group and in the $\mathrm{DZ}$ group, but found that as they grew older, the pairs of $M Z$ twins became more similar than DZ twins. A similar trend was also reported who showed that the intrapair variance for different phenotypes such as vital capacity, vertical jump and heart rate was lower in MZ twins when compared with DZ twins (Chatterjee \& Das, 1995).

Twins and/or multiple birth siblings provide an excellent opportunity to study interindividual variability of developmental plasticity in neuromotor performance. Twins share the same maternal environment, however, its influence may differently affect both fetuses, because each one has its own fetoplacental environment. Because $\mathrm{MZ}$ share the same set of genes identical by descent, the association between intra-pair differences in birth size and adult outcomes are due, mainly, to environmental differences (including fetoplacental influences) (Morley et al., 2003). It has to be acknowledged that assessing and estimating neuromotor performance or motor coordination functions in children and adolescents are challenging tasks given the difficulty in finding coherent test batteries that are easily applied, clinically sound and relevant, psychometrically valid and reliable where normative data for a wide range of ages is provided. Three recent articles (Heineman \& Hadders-Algra, 2008; Wouter et al., 2009; Lopes et al., 2011) presented different alternatives to neuromotor and/or gross motor coordination assessment in infants, children and adolescents. Despite their methodological diversity and distinct scoring systems, all validly highlight changes in fundamental motor skills and gross motor coordination in children with and without LBW. Notwithstanding these different possibilities, the ZNA test battery used in the present study was based upon sound research with a particular emphasis in school studies and clinical pediatrics, which were associated to a sound and robust methodological foundation (Largo et al., 2003). The ZNA test battery has proven its reliability and validity, and that reliability in this study was also very acceptable.

The present study has some limitations that should be acknowledged. The first one refers to the small sample size with its statistical power limitations (Bouchard et al., 1997), which is related to the time-consuming procedure in assessing the neuromotor ZNA test itself and motor component scoring using filmed material. Other twin studies with a focus on motor coordination/ neuromotor performance are also limited in sample size. For example, one study used $15 \mathrm{MZ}$ males, $11 \mathrm{DZ}$ males, $12 \mathrm{MZ}$ females and DZ females to study within-pair variance in kinematic aspects of inter-limb motor coordination in a 60-m dash (Sklad, 1972).

When dealing with small twin sample sizes and in order to increase statistical power, it has been suggested to deal only with MZ and DZ twins regardless of their sex (Bouchard et al., 1997). In the present study, given the sex differences in age and sport activities, removing their effect allowed us to refine the phenotypes. The second limitation relates to the lack of more precise and extensive information regarding systematic sports participation of each twin, as it might more extensively covary with all motor tasks. Extensive practice might remove/overcome the magnitude of birth weight effects on the twins' neuromotor performance. A third limitation concerns the small number of subjects with LBW. However, this study has also important strengths that need to be highlighted, including the use of DNA for zygosity determination and the use of a coherent, valid and reliable test battery to assess neuromotor performance (Largo et al., 2003).

In conclusion, our study showed: (a) limited influence of birth weight on neuromotor performance of children and adolescents; (b) a higher proportion of twins' neuromotor performance values below percentile 10; (c) the performance of the quality of movements are very low, 
Neuromotor performance in twins

suggesting that twins may not develop similar strategies to enable them to achieve the same neuromotor "levels" as the referred values in relation to the time of performance; and (d) a greater homogeneity in neuromotor performance in MZ compared with DZ twins, indicative for the influence of a genetic component.

\section{Perspectives}

It is well documented that, at the population level, general motor performance exhibits a wide range of scores according to sex and age; a similar pattern is evident in gross motor coordination, and more so in neuromotor performance. Children of the same age and sex may differ in their neuromotor performance for many reasons, among those also their birth weight. Physical education teachers and coaches have to be aware of this knowledge to identify this possible reason for lower performance and plan specific training programs and/or organize their class exercises and sports demands to match this adverse condition. Likewise, a similar strategy has to be developed when physical education teachers and/or coaches deal with twins given their propensity for lower neuromotor performance.

Key words: low birth weight, neuromotor performance, youth, twins.

\section{References}

Akerman BA, Fischbein S. Within-pair similarity in $\mathrm{MZ}$ and $\mathrm{DZ}$ twins from birth to eighteen years of age. Acta Genet Med Gemellol (Roma) 1992: 41: 155-164.

Barker DJ. Fetal origins of coronary heart disease. Acta Paediatr Suppl 1997: 422: 78-82.

Bouchard C, Malina RM, Pérusse L. Genetics of fitness and physical performance. Leeds and Champaign, IL: Human Kinetics Publisher, 1997: 89-98.

Brun CC, Leporé N, Pennec X, Lee AD, Barysheva M, Madsen SK, Avedissian C, Chou YY, de Zubicaray GI, McMahon KL, Wright MJ, Toga AW, Thompson PM. Mapping the regional influence of genetics on brain structure variability - a tensor-based morphometry study. Neuroimage 2009: 48 (1): 37-49.

Chatterjee S, Das N. Physical and motor fitness in twins. Jpn J Physiol 1995: 45 (3): 519-534.

Escobar GJ, Clark RH, Greene JD. Short-term outcomes of infants born at 35 and 36 weeks gestation: we need to ask more questions. Semin Perinatol 2006a: 30 (1): 28-33.

Escobar GJ, McCormick MC, Zupancic JA, Coleman-Phox K, Armstrong MA, Greene JD, Eichenwald EC, Richardson DK. Unstudied infants: outcomes of moderately premature infants in the neonatal intensive care unit. Arch Dis Child Fetal Neonatal Ed 2006b: 91 (4): 238-244.

Evensen KA, Vik T, Helbostad J, Indredavik MS, Kulseng S, Brubakk AM. Motor skills in adolescents with low birthweight. Arch Dis Child Fetal Neonatal Ed 2004: 89 (5): 451-455.

Hack M, Taylor HG, Klein N, Mercuri-Minich N. Functional limitations and special health care needs of 10 to 14 years old children weighing less than $750 \mathrm{~g}$ at birth. Pediatrics 2000: 106 (3): 554-560.

Hajnal BL, Braun-Fahrländer C, von Siebenthal K, Bucher HU, Largo RH. Improved outcome for very low birthweight multiple births. Pediatr Neurol 2005: 32 (2): 87-93.

Heineman KR, Hadders-Algra M. Evaluation of neuromotor function in infancy - a systematic review of available methods. J Dev Behav Pediatr 2008: 29 (4): 315-323.

Jongmans M, Mercuri E, de Vries L, Dubowitz L, Henderson SE. Minor neurological signs and perceptual-motor difficulties in prematurely born children. Arch Dis Child Fetal Neonatal Ed 1997: 76 (1): 9-14.

Lareu MV, Phillips CP, Carracedo A, Lincoln PJ, Syndercombe Court D, Thomson JA. Investigation of the STR locus $\mathrm{HUMTH}_{01}$ using PCR and two electrophoresis formats: UK and Galician Caucasian population surveys and usefulness in paternity investigations. Forensic Sci Int 1994: 66 (1): 41-52.

Largo R, Fischer J, Caflisch J. eds. Handbook of Zurich Neuromotor Assessment. Zurich: Department of Growth and Development, University Children's Hospital, 2002: 10-80.

Largo RH, Caflisch JA, Hug F, Muggli K, Molnar AA, Molinari L, Sheehy A, Gasser ST. Neuromotor development from 5 to 18 years: part 1 and 2: time performance and associated movements. Dev Med Child Neurol 2001: 43 (7): 436-453.

Largo RH, Fischer JE, Rousson V. Neuromotor development from kindergarten age to adolescent: developmental course and variability. Swiss Med Wkly 2003: 133: 193-199.
Loos RJ, Beunen G, Fagard R, Derom C, Vlietinck R. Birth weight and body composition in young women: a prospective twin study. Am J Clin Nutr 2002: 75 (4): 676-682.

Lopes AAT, Go T, Maia JAR.

Neuromotor performance, prematurity and low birth weight. Rev Bra Cineantropom Desempenho Hum 2011: 13 (1): 73-81.

Maia J, Lopes V, Seabra A, Garganta R. Genetic and environmental effects in physical activity and health related physical fitness. A study in twins aged 6-to-12 years of the Azores Islands (Portugal). R Bras Ci e Mov 2003: 11 (4): 33-44.

Morley R, Dwyer T, Carlin JB. Studies of twins: can they shed light on the fetal origins of adult disease hypothesis. Twin Res 2003: 6 (6): 520-525.

Msall ME, Tremont MR. Measuring functional outcomes after prematurity: developmental impact of very low birth weight and extremely low birth weight status on childhood disability. Ment Retard Dev Disabil Res Rev 2002: 8 (4): 258-272.

Palta M, Sadek-Badawi M, Evans M, Weinstein MR, McGuinnes G. Functional assessment of a multicenter very low birthweight cohort at age 5 years. Arch Pediatr Adolesc Med 2000: 154 (1): 23-30.

Rogers M, Fay TB, Whitfield MF, Tomlinson J, Grunan RE. Aerobic capacity, strength, flexibility, and activity level in unimpaired extremely low birthweight $(<800 \mathrm{~g})$ survivors at 17 years of age. Compared with term-born control subjects. Pediatrics 2005: 116 (1): 58-65.

Russell RB, Green NS, Steiner CA, Meikle S, Howse JL, Poschman K. Cost of hospitalization for preterm and 


\section{Lopes et al.}

low birth weight infants in the United States. Pediatrics 2007: 120: 1-9.

Saigal S, Stoskopf BL, Streiner DL, Burrows E. Physical growth and current health status of infants who were of extremely low birth weight and controls at adolescence. Pediatrics 2001: 108 (2): 407-415.

Saigal S, Szatmari P, Rosenbaum P, Campbell D, King S. Intellectual and functional status at school entry of children who weighed 1000 grams or less at birth: a regional perspective of births in the 1980s. J Pediatr 1990: 116 (3): 409-416.

Seitz J, Jenni OG, Molinari L, Caflisch J, Largo RH, Latal Hajnal B. Correlations between motor performance and cognitive functions in children born $<1250 \mathrm{~g}$ at school age. Neuropediatrics 2006: 37 (1): 6-12.

Sklad M. Similarity of movement in twins. Wychowanie Fizycznie I Sport 1972: 16: 119-141.

Walther FJ, den Ouden AL,

Verloove-Vanhorick SP. Looking back in time: outcome of a national cohort of very preterm infants born in The Netherlands in 1983. Early Hum Dev 2000: 59 (3): 175-191.

Wardlaw T, Blanc A, Zupan J, Åhman E. Low Birthweight Country, Regional and Global Estimates. New York: The
United Nations Children's Fund and World Health Organization, UNICEF and WHO, Editorial and Publications, 2004: 1-9.

Wolf MJ, Koldewijn K, Beelen A, Smit B, Hedlund R, de Groot IJ.

Neurobehavioral and developmental profile of very low birthweight preterm infants in early infancy. Acta Paediatr 2002: 91 (8): 930-938.

Wouter C, Kristine De M, Christiane S, Caroline A. Movement skill assessment of typically developing preschool children: a review of seven movement skill assessment tools. J Sports Sci Med 2009: 8: 154-168. 